\title{
CRITERIOS URBANOS Y ARQUITECTÓNICOS INMATERIALES EN EL PLAN URBANO MODERNO DE GUENGA DE 1947
}

\author{
IMMATERIAL URBAN AND ARGHITEGTURAL \\ GRITERIA IN GUENGA'S 1947 MODERN URBAN PLAN
}

(). $(1)(\mathbb{N}$

Arquitecto por la Universidad de Cuenca en 2005. Diplomado superior en Gerencia de Gobiernos Seccionales por la Universidad del Azuay en 2008. Magíster en Proyectos Arquitectónicos por la Facultad de Arquitectura de la Universidad de Cuenca en 2010. Ganador del concurso de méritos y oposición a nivel nacional para la coordinación de bienes materiales de la Regional 3 del Instituto Nacional de Patrimonio Cultural (2010-2011); ha sido conferencista por el centro de postgrados de la Facultad de arquitectura y urbanismo de la Universidad de Cuenca y do.co.mo.mo.- capitulo ecuador (2012). Docente universitario de tercero y cuarto nivel por la Universidad Técnica de Ambato (2011-2013). Docente universitario por la Universidad Tecnológica Indoamérica (2014-2015). Actualmente se desempeña como Docente titular y director de proyectos de investigación por la Universidad Nacional de Chimborazo; y es gerente de la oficina y consultora de arquitectura "muy\&asociados".

nmuy@unach.edu.ec

orcid.org/0000-0002-0335-7668 


\section{Pedro Samaniego \\ Universidad del Azuay \\ Ecuador}

Graduado de Arquitecto en la Universidad de Cuenca (Ecuador) Agosto 2003. Magíster en "Proyectos Arquitectónicos" en la Universidad de Cuenca (Ecuador). Febrero 2008. Tesis de maestría: "La Facultad de Arquitectura de la Universidad de Cuenca, Álvaro Malo C. Cuenca - Ecuador, 1973 - 77". Fue recomendada para publicación. Profesor de Proyectos Arquitectónicos de Escuela de Arquitectura de la Universidad del Azuay, desde septiembre 2011 hasta la fecha. Profesor de maestrías en proyectos arquitectónicos. Profesor invitado a varias Universidades. Investigador con varias publicaciones en revistas científicas. Publicaciones en varias revistas de arquitectura. Primer premio Nacional en la Bienal de Arquitectura de Quito XVII, en la categoría de diseño arquitectónico, con las Casas Samaniego. Año 2010. Premio otorgado: Ciudades Sostenibles y Asentamientos Humanos, Cerro Verde Ecolodge y Centro de Investigación Galápagos Organic Orgal S.A. en el $11^{\circ}$ Foro Mundial sobre Asentamientos Humanos en el marco de HABITAT III, celebrado en Quito-Ecuador, año 2016. Director de Planeamiento de la Universidad del Azuay. Gerente de la oficina de Arquitectura y Diseño AyD.

pjsamaniego@uazuay.edu.ec orcid.org/0000-0002-3076-8390

Fecha de recepción: 06 de marzo, 2020. Aceptación: 19 de octubre, 2020. 


\section{Resumen}

El estudio analiza criterios urbanísticos y arquitectónicos inmateriales del plan urbano moderno de la ciudad de Cuenca, Ecuador, desarrollado en 1947 por el arquitecto uruguayo Gilberto Gatto Sobral. El trabajo comprende tres momentos: 1) Investigación descriptiva de la influencia que tuvo el arquitecto para desarrollar el plan regulador de Cuenca. 2) Revisión de casos de estudio de los planes normativos y urbanísticos que se han desarrollado a nivel nacional e internacional, y 3) Investigación exploratoria donde se toma como punto de partida el plan del arquitecto Gatto Sobral en Cuenca, para realizar una serie de hipótesis sobre la proporción, la escala y la traza reguladora. El estudio concluye con criterios de forma urbana y arquitectónica a través de tres ideas principales: El módulo como entidad delimitante original (criterio clásico como la proporción). La valoración esencial del proceso histórico (huella original como medio de crecimiento urbano), y la condición de la forma urbana de la Cuenca de 1947. Resultados que pretenden aportar a la ciudad, y poner en valor histórico a la cultura de la modernidad.

\section{Palabras clave}

Criterios urbanos y arquitectónicos, inmaterial, valor patrimonial, plan urbano moderno.

\section{Abstract}

The study analyzes immaterial urban and architectural criteria of the modern urban plan of the city of Cuenca, Ecuador, developed in 1947 by the Uruguayan architect Gilberto Gatto Sobral. The work comprises three moments: 1) Descriptive investigation of the influence that the architect Gatto Sobral had to develop Cuenca's regulatory plan. 2) Review of case studies of the regulatory plans and urban plans that have been developed at the national and international level, and 3) Exploratory research where the plan of the architect Gatto Sobral in Cuenca is taken as a starting point, to make a series of hypotheses about the proportion, the scale, and the regulatory trace. The study concludes with criteria of urban and architectural form through three main ideas: the module as an original delimiting entity (the classic criterion like proportion), the essential assessment of the historical process (original trace as a means of urban growth), and the condition of the urban form of Cuenca from 1947. The results intend to contribute to the city and to give historical value to modern culture.

\section{Keywords}

Urban and architectural criteria, immaterial, heritage value, modern urban plan. 


\section{Introducción}

Para el presente caso se pretende una inmersión en el anteproyecto del Plan Regulador de Cuenca 1947, realizado por el arquitecto Gilberto Gatto Sobral con el fin de indagar si su intervención presenta criterios $^{1}$ no visibilizados.

Como herramientas se han planteado investigaciones descriptivas, exploratorias y estudio de casos; estas serán abordadas desde la arquitectura y el urbanismo, y apuntarán a entender el proceso formativo del autor hasta la proyectación del plan para Cuenca. Las intervenciones, urbanas y arquitectónicas, realizadas dentro de una línea de tiempo anterior a la que vivimos, mostrarán, si las observaciones, 0, críticas son realizadas de manera superficial. Es pertinente indicar que el presente artículo recibe el apoyo de la investigación ejecutada por Muy y Samaniego (2009), y que conjuntamente con lo que se está planteando, pueden coadyuvar a la toma de decisiones por parte de organismos públicos y privados, encargados de áreas patrimoniales, urbanas y arquitectónicas.

\section{Materiales / Métodos}

En el título del documento se usan estas palabras "criterios inmateriales"; por lo que es necesario definir la palabra "criterio" como un juicio 0 discernimiento2, que todo ser humano lo usa para tomar decisiones, y en cuanto a la palabra "inmaterial"3 se la entenderá como algo que no puede ser percibido por los sentidos.

Discernidas las palabras anteriores se condiciona que esta investigación estará delimitada por criterios formales 0 actos figurativos de intervención frente a una preexistencia histórica. Con esta perspectiva, es necesario entender la evolución del Arq. Gatto Sobral, en cuanto su formación e influencias, se inicia este recorrido indicando, lo que para él, es la arquitectura y el urbanismo, para esto se transcribe lo que Maldonado4 (1991), extrae de la revista Trama N ${ }^{\circ}$ 50:
En cierta ocasión, hablando ante un grupo de alumnos, Gatto Sobral expresó una idea que resume su criterio arquitectónico: Dijo que la Arquitectura y el urbanismo son 2 aspectos de una misma disciplina, la organización del espacio para el uso del hombre. La diferencia está solamente en la escala: El Urbanismo es Arquitectura en gran escala, mientras que la Arquitectura es un Urbanismo de detalle (p.11).

Con esta posición se establece que, para Gatto Sobral, hablar de la "disciplina de la arquitectura" es abordar el tema urbano y arquitectónico al mismo tiempo. La información precedente, presupone que Gatto Sobral tuvo una formación nutrida en la Historia y Teoría de la Arquitectura previo a su intervención en Cuenca, para corroborar estas aseveraciones se ha implementado una metodología

\footnotetext{
${ }^{1}$ Criterio: Norma para conocer la verdad. (RAE,2001, https://n9.cl/tyqf6).

${ }^{2}$ Discernimiento: Distinguir algo de otra cosa, señalando la diferencia que hay entre ellas. Comúnmente se refiere a operaciones del ánimo. (RAE,2001, https://n9.cl/mxfr).

${ }^{3}$ Inmaterial: no material. (RAE,2001, https://n9.cl/mvqye)

${ }^{4}$ Maldonado, C. (1991). Gilberto Gatto Sobral en la arquitectura ecuatoriana. Revista Arquitectura No 261; Sociedad de Arquitectos de Uruguay. Montevideo, Uruguay: editorial Escuelas Profesionales Talleres Don Bosco
} 
subdividida en tres momentos: El primero, que está delimitado por investigaciones descriptivas, en la que por medio de matrices que contienen líneas de tiempo generales (Matriz 1. Influencias en la formación de Gatto Sobral / Matriz 2. Antigüedad - traza concéntrica/ / Matriz 3. Carta de Atenas - Memoria descriptiva del Plan Urbano para Cuenca), se obtendrán relaciones cognitivas y pragmáticas de la historia y teoría del arquitecto, previo a su anteproyecto. El segundo que corresponde a investigaciones por estudios de casos, en la que se atiende a la investigación dada por Muy y Samaniego (2009), acá, se muestran casos locales de intervención en planes urbanos (Pelileo, Píllaro), también se hace un acercamiento al caso Bogotá; el tercero se basa en los dos anteriores ya que permite investigaciones exploratorias, a través, de redibujo (reconstrucciones digitales), estas acciones permitirán entender con mayor precisión la actuación del Arq. Gatto Sobral al momento de concebir el anteproyecto.

\section{Primer momento: Investigación descriptiva}

Este segmento resulta de la interacción de tres matrices, resueltas todas, permitirá un acercamiento al nivel de formación del Arq. Gatto Sobral. Como punto de partida se toma la evidencia que Maldonado4 (1991), extrae de la revista Trama № 50, describiendo que:

Gatto Sobral, aplicaría los principios de diseño derivados de su formación con algunos destacados maestros uruguayos como Vilamajó y Gómez Gavazzo, y bajo la influencia de otros grandes maestros franceses, como Guadet, Gromort y Perret (Gatto Sobral todavía no tenía mucha simpatía ni confianza en las ideas de Le Corbusier ni de Wright) (p.11).
Ante esta evidencia es ineludible que la primera matriz a trabajar es una línea de eventos generales que ubique a cada maestro de Gatto Sobral en el tiempo (Anexo Tabla 1).

Como datos relevantes de esta matriz, se obtiene:

a) Que, en relación a la historia, referencia sus estudios según pautas clásicas de la Escuela de bellas artes de París, lo confirman cuatro de los seis en análisis; b) Que tiene conocimiento sobre el movimiento moderno y se lo debe a cuatro de los seis nombres expuestos; c) Que tiene conocimiento sobre Planeamiento Urbano y se lo debe a la formación de los seis arquitectos, ya que cada uno desde sus frentes demuestran ser críticos con respecto a la historia y teoría de la arquitectura; d) Que tiene conocimiento y conciencia sobre el hecho de dar valor a las preexistencias (Patrimonio), lo confirman con sus actuaciones los 6 maestros influyentes. De este análisis, se desprende que Gatto Sobral proviene de una formación mixta, la neoclásica francesa y la moderna (influencia CIAM, Le Corbusier). 
Tabla 1. Matriz de Influencias de Gilberto Gatto Sobral

\begin{tabular}{|c|c|c|c|c|c|c|}
\hline \multicolumn{7}{|c|}{ MATRIZ \# 1 - INFLUENCIAS GILBERTO GATTO SOBRAL } \\
\hline PAIS & \multicolumn{2}{|c|}{ URUGUAY } & \multicolumn{3}{|c|}{ FRANCIA } & \multirow{2}{*}{$\begin{array}{l}\text { FRANCO - SUIZO } \\
\text { Le Corbusier } \\
(1887-1965)\end{array}$} \\
\hline Nombre & $\begin{array}{l}\text { Vilamajó } \\
(1894-1948)\end{array}$ & $\begin{array}{l}\text { Gomez Gavazzo } \\
\text { (1904-1987) }\end{array}$ & $\begin{array}{l}\text { Julien Guadet } \\
(1834 \text { - 1908) }\end{array}$ & $\begin{array}{l}\text { Georges Gromort } \\
(1870-1961)\end{array}$ & $\begin{array}{l}\text { Auguste Perret } \\
(1874 \text { - 1954) }\end{array}$ & \\
\hline : & $\begin{array}{l}\text { L' Ecole des Beaux-Arts } \\
\text { (Pautas clásicas), en una } \\
\text { época de transición hacia } \\
\text { la arquitectura moderna. }\end{array}$ & $\begin{array}{l}1933 \text { trabaja con Le } \\
\text { Corbusier (movimiento } \\
\text { moderno). }\end{array}$ & $\begin{array}{l}\text { Se forma en la L' Ecole } \\
\text { des Beaux-Arts. / Apego } \\
\text { al clasicismo. }\end{array}$ & $\begin{array}{l}\text { L'Ecole des Beaux-Arts./ } \\
\text { Sus libros de teoría } \\
\text { siguen a los de Julien } \\
\text { Guadet. }\end{array}$ & $\begin{array}{l}\text { L' Ecole des Beaux-Arts./ } \\
\text { Mayor representante del } \\
\text { nuevo Clasicismo francés, } \\
\text { queaparece a comienzos } \\
\text { del s. XX. }\end{array}$ & $\begin{array}{l}\text { No se gradúa en ninguna } \\
\text { universidad,./ Recorre } \\
\text { múltiples lugares para } \\
\text { aprender }\end{array}$ \\
\hline  & $\begin{array}{l}\text { 1. Admira obras clásicas y } \\
\text { Renacentistas, en 1934, } \\
\text { toma partida por el } \\
\text { movimiento moderno. } \\
\text { 2. Proyecta la Sede ONU, } \\
\text { en equipo con Le } \\
\text { Corbusier y Oscar } \\
\text { Niemeyer. } \\
\text { 3. Manifiesta que... "el } \\
\text { trabajo bien heche está } \\
\text { definido desde tiempo } \\
\text { antiguo, como una } \\
\text { alianza de lo bello, lo } \\
\text { cómodo, lo sólido y lo } \\
\text { económico... }\end{array}$ & 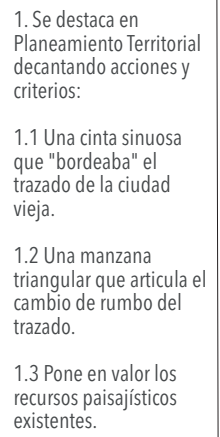 & $\begin{array}{l}\text { 1. Define a la arquitectu- } \\
\text { ra desde la función así... } \\
\text { "la puesta en obra, para } \\
\text { satisfacer las necesida- } \\
\text { des materiales y morales, } \\
\text { de los elementos de la } \\
\text { construcción". } \\
\text { 2. Restaura el Théatre- } \\
\text { Francais, con esto marca } \\
\text { un determinante respeto } \\
\text { al pasado. }\end{array}$ & $\begin{array}{l}\text { 1. Publica sobre la } \\
\text { antigüedad, renacimien- } \\
\text { to y modernidad. } \\
\text { 2. Manifiesta que es, } \\
\text { indispensable estudiar } \\
\text { las arquitecturas } \\
\text { precedentes para } \\
\text { proseguir la tradición. } \\
\text { 3. El urbanismo es el } \\
\text { "sumum" de la arquitec- } \\
\text { tura; no se puede } \\
\text { concebir que un } \\
\text { urbanista no sea primero } \\
\text { arquitecto. }\end{array}$ & $\begin{array}{l}\text { 1. Manifiesta un retorno } \\
\text { a la tradición clásica, } \\
\text { incluyendo la materia } \\
\text { hormigón en sus } \\
\text { obras. } \\
\text { 2. Dice que las necesida- } \\
\text { des se satisfacen con el } \\
\text { empleo juicioso de la } \\
\text { materia, según las leyes } \\
\text { de la economía. También } \\
\text { dice que todo verdadero } \\
\text { arquitecto es urbanista. }\end{array}$ & $\begin{array}{l}\text { 1. Define al urbanismo } \\
\text { como ciencia de } 3 \\
\text { dimensiones: Espacio, } \\
\text { Suficiente sol y } \\
\text { ventilación, altura. } \\
\text { 2. En la carta de Atenas } \\
\text { habla sobre el respeto a } \\
\text { las ciudades históricas. } \\
\text { 3. En } 1938 \text { dice que la } \\
\text { tradición.... es hacia } \\
\text { "adelante" y no hacia } \\
\text { "atrás". También } \\
\text { habla que la arquitectura } \\
\text { nueva se manifiesta, al } \\
\text { volver a valorar la escala } \\
\text { humana. }\end{array}$ \\
\hline CITA & \multicolumn{6}{|c|}{$\begin{array}{l}\text { https://es.wikipedia.org/wiki/Julio_Vilamajó/ https://es.wikipedia.org/wiki/Carlos_Gómez_Gavazzo/ http://www.rochainfo.com/balneario/urpal.htm)/ Diccionario Akal de la } \\
\text { Arquitectura del siglo XX Por Jean-Paul Midant/ https://fr.wikipedia.org/wiki/Georges_Gromort/ https://issuu.com/bsau/docs/1939-200/ https://es.wikipedia.org/wiki/Augus- } \\
\text { te_Perret/ Cohen, Jean-Louis (2006) Le Corbusier 1887-1965. El lirismo de la arquitectura en la era mecánica, Ed. Taschen, Bremen, 96 pp. ISBN 978-3-8228-3533-3/ Le } \\
\text { Corbusier (1943) La Charte d'Athènes (ed.castellano Principios de urbanismo. La Carta de Atenas, Ed. Planeta, 1993, Barcelona, 151 pp. ISBN 84-395-2188-X). }\end{array}$} \\
\hline
\end{tabular}

Dados los datos generales precedentes y enfocando que lo que se busca es un acercamiento a la manera en que Gatto Sobral proyectó el plan urbano, es necesario generar una línea de tiempo basado en trazas urbanas, para esto se genera la matriz número dos, que toma como referencia a Samper ${ }^{5}$ (1997), por medio de uno de sus libros (Recinto Urbano "la humanización de la ciudad") (Anexo Tabla 2). 
Tabla 2. Matriz de trazas urbanas, desde la reticular a la concéntrica

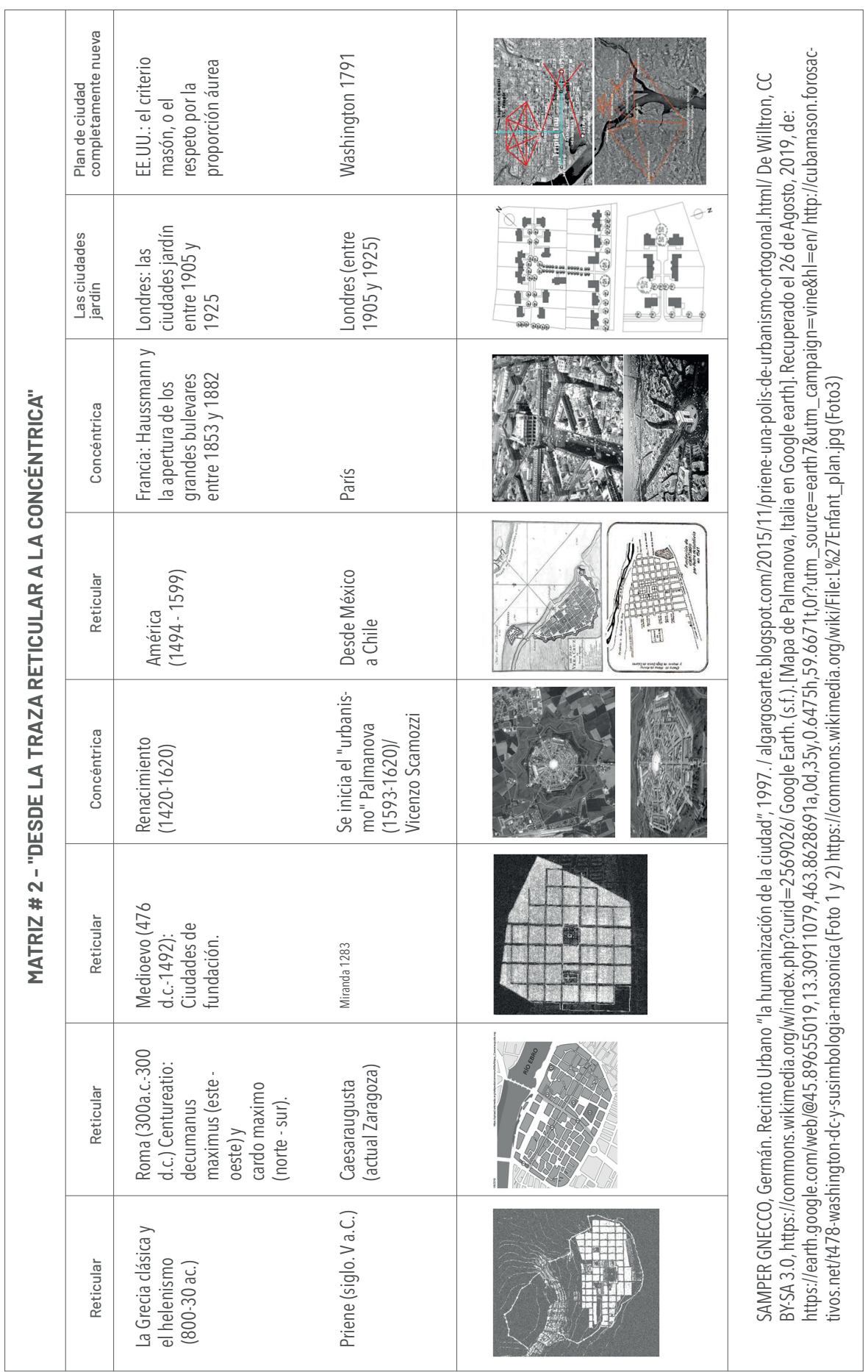


Al comparar visualmente el redibujo del anteproyecto del plan regulador de 1947, realizado por Muy y Samaniego (2009), con la matriz número dos, que contiene el recorrido histórico de las trazas, se identifica que la ciudad presenta múltiples decisiones técnicas para ordenar la forma urbana, incluso segmentando una actuación para cada época mencionada, así:

e) La ciudad histórica (Cuenca) presenta la retícula ortogonal como medio de fundación y organización urbana, misma que ha sido utilizada desde la antigüedad clásica (Grecia 800-30 a.C. / Roma 300 a.C.-300 d.C.), Medioevo (476 d.C.-1492 ciudades de fundación), América (fundaciones 1494-1599). f) El lugar de emplazamiento de la nueva ciudad (actual Ejido) presenta consideraciones urbanas como las trazas concéntricas, como ejemplo citamos a las utilizadas en el Renacimiento (1420-1620) por Vicenzo Scamozzi en Palmanova, Italia (1593-1620) y en París por su prefecto Georges Haussmann (18531882). g) Las recomendaciones del anteproyecto del plan regulador de 1947 en relación a vivienda, tienen que ver con las ciudades jardín, tan estudiadas y aplicadas en Londres (1905-1925). Aunque la información de estas dos matrices, no indican contundencias, si explican de manera asertiva que Gatto Sobral contenía conocimiento previo sobre la disciplina arquitectónica, "garantizando juicio antes de la toma de decisiones". La matriz número tres, tiene que ver con las relaciones arquitectónicas, urbanas y valoración a la preexistencia, que proponen, tanto, la carta de Atenas (CIAM), como la memoria descriptiva del anteproyecto del plan regulador de Cuenca 1947. El resultado obtenido permite establecer similitudes, así en el ámbito de arquitectura presenta un alto grado, en el ámbito urbano muy alto y en el ámbito patrimonial medio. 
Tabla 3. Matriz de relación entre el CIAM y el plan regulador de Cuenca

\begin{tabular}{|c|c|c|c|c|c|c|c|}
\hline \multicolumn{8}{|c|}{ MATRIZ \# 3 - RELACION ENTRE CIAM Y MEMORIA DESCRIPTIVA DEL ANTEPROYECTO DEL PLAN REGULADOR CUENCA 1947.} \\
\hline \multicolumn{8}{|c|}{$\begin{array}{l}\text { Se muestran } 17 \text { criterios esenciales (fijos) de la carta de Atenas (CIAM), en las áreas de arquitectura, urbanismo y patrimonio, con fines de compararlos con los que expone la memoria descriptiva del } \\
\text { anteproyecto del plan regulador de } 1947 \text { para Cuenca. En la columna "SUMAAPRC", se sumara lo símil o restara lo no símil de derecha a izquierda o de arriba hacia abajo; el resultado mostrará el porcenta- } \\
\text { je de coincidencias en sus planteamientos. }\end{array}$} \\
\hline \multicolumn{3}{|c|}{ LE CORBUSIER } & \multirow{2}{*}{$\begin{array}{l}\text { SUMA } \\
\text { CIAM }\end{array}$} & \multirow{2}{*}{$\begin{array}{l}\text { SUMA } \\
\text { APRC }\end{array}$} & & \multirow{2}{*}{$\begin{array}{l}\text { GILBERTO GATTO SOBRAL } \\
\text { URBANISMO }\end{array}$} & \multirow[b]{2}{*}{ ARQUITECTURA } \\
\hline ARQUITECTURA & URBANISMO & PATRIMONIO & & & PATRIMONIO & & \\
\hline \multicolumn{3}{|c|}{ Considera al hombre como unidad de medida (modulor) } & & & \multicolumn{3}{|c|}{ Prioriza a hombre como origen de toda organización superior } \\
\hline $\begin{array}{l}\text { La vivienda debe tener } \\
\text { primacía sobre el resto } \\
\text { de usos. }\end{array}$ & $\begin{array}{l}\text { Se basa en la } \\
\text { zonificacion }\end{array}$ & $\begin{array}{l}7 \text { Las razones que presiden el } \\
\text { desarrollo de las ciudades } \\
\text { están, pues, sometidas a } \\
\text { cambios continuos. }\end{array}$ & 3 & 2 & $\begin{array}{l}\text { No se encuentran } \\
\text { similitudes }\end{array}$ & $\begin{array}{l}\text { Se basa en la } \\
\text { zonificacion }\end{array}$ & $\begin{array}{l}\text { Contempa el tipo una } \\
\text { vivienda un arbol, } \\
\text { apreciable en los cortes } \\
\text { que muestran las secciones } \\
\text { de vias planteadas. }\end{array}$ \\
\hline $\begin{array}{l}\text { En la situación de la } \\
\text { residencia se buscará la } \\
\text { higiene. }\end{array}$ & $\begin{array}{l}\text { Función habitar } \\
\text { (vivienda) }\end{array}$ & $\begin{array}{l}24 \text { La determinación de las } \\
\text { zonas de habitación debe estar } \\
\text { dictada por razones de higiene. }\end{array}$ & 3 & 2 & $\begin{array}{l}\text { No se encuentran } \\
\text { similitudes }\end{array}$ & $\begin{array}{l}\text { Función habitar (Contem- } \\
\text { pla al hombre como } \\
\text { núcleo de la } \\
\text { sociedad) }\end{array}$ & $\begin{array}{l}\text { Principios higiénicos en la } \\
\text { ubicación } \\
\text { de actividad, trabajo, } \\
\text { comercio y } \\
\text { residencia. }\end{array}$ \\
\hline $\begin{array}{l}\text { La relación vivienda/su- } \\
\text { perficie la determinan } \\
\text { las características del } \\
\text { terreno en función del } \\
\text { asoleamiento. }\end{array}$ & Función trabajar & $\begin{array}{l}40 \text { Deben estimarse los } \\
\text { elementos existentes: ríos, } \\
\text { bosques, colinas, montañas, } \\
\text { valles, lago, mar, etc. }\end{array}$ & 3 & 3 & $\begin{array}{l}\text { Propone una ordenanza } \\
\text { que contempla la estética } \\
\text { paisajística y arquitectónica. }\end{array}$ & Función trabajar & $\begin{array}{l}\text { En las zonas se tendrá más } \\
\text { o menos } \\
\text { una densidad de } 75 y \\
\text { 25Hab./ha. }\end{array}$ \\
\hline $\begin{array}{l}\text { Se debe prohibir la } \\
\text { disposición de viviendas } \\
\text { a lo largo de vías de } \\
\text { comunicación. }\end{array}$ & $\begin{array}{l}\text { Función recrear(el } \\
\text { cuerpo y el espíritu: } \\
\text { salud, educación, } \\
\text { esparcimiento, etc.) }\end{array}$ & $\begin{array}{l}57 \text { Los trazados suntuarios, con } \\
\text { finalidad representativa, han } \\
\text { podido o pueden constituir } \\
\text { graves dificultades para la } \\
\text { circulación. }\end{array}$ & 3 & 2 & $\begin{array}{l}\text { Propone arterias de } \\
\text { circulación internas y } \\
\text { externas a la urbe, } \\
\text { manteniendo las condicio- } \\
\text { nes naturales de su } \\
\text { topografía y por tanto su } \\
\text { paisaje. Incorpora nuevas } \\
\text { áreas ampliando la } \\
\text { sección de vías y veredas } \\
\text { para futuras } \\
\text { necesidades. }\end{array}$ & Función recrear & $\begin{array}{l}\text { No se encuentran } \\
\text { similitudes }\end{array}$ \\
\hline \multirow[t]{3}{*}{$\begin{array}{l}\text { La solución son las } \\
\text { viviendas en altura } \\
\text { situadas a una distancia } \\
\text { entre ellas que permite } \\
\text { la construcción de } \\
\text { grandes superficies } \\
\text { verdes. }\end{array}$} & $\begin{array}{l}\text { Función circular } \\
\text { (conectar las otras } \\
3 \text { entre sí) }\end{array}$ & $\begin{array}{l}65 \text { Los valores arquitectónicos } \\
\text { deben ser salvaguardados } \\
\text { (edificios aislados o conjuntos } \\
\text { urbanos). }\end{array}$ & 3 & 3 & $\begin{array}{l}\text { Propone una ordenanza } \\
\text { que contempla la estética } \\
\text { paisajística y arquitectónica. } \\
\text { Considera inventariar el } \\
\text { valor de la propiedad } \\
\text { privada, pública y el } \\
\text { equipamiento municipal. }\end{array}$ & $\begin{array}{l}\text { Función circular . Trazado } \\
\text { de arterias de comunica- } \\
\text { ción (externas e internas). }\end{array}$ & $\begin{array}{l}\text { Establece ordenar, localizar } \\
\text { y proporcionar las } \\
\text { funciones activas y } \\
\text { pasivas para la colectividad } \\
\text { a través del recurso de la } \\
\text { zonificación. }\end{array}$ \\
\hline & & $\begin{array}{l}66 \text { Los testimonios del pasado } \\
\text { serán salvaguardados si son } \\
\text { expresión de una cultura } \\
\text { anterior y si responden a un } \\
\text { interés general. }\end{array}$ & 1 & 1 & $\begin{array}{l}\text { La relación vivienda/super- } \\
\text { ficie la determinan el } \\
\text { crecimiento a partir de la } \\
\text { primitiva ciudad con } \\
\text { tendencia al oeste y al sur } \\
\text { con los márgenes de los } \\
\text { caminos. }\end{array}$ & & \\
\hline & & $\begin{array}{l}67 \text { Si su conservación no } \\
\text { implica el } \\
\text { sacrificio de poblaciones } \\
\text { mantenidas en condiciones } \\
\text { malsanas. }\end{array}$ & 1 & 0 & $\begin{array}{l}\text { No se encuentran } \\
\text { similitudes }\end{array}$ & & \\
\hline
\end{tabular}


Los porcentajes generados por medio de identificar los objetivos de la carta y la memoria descriptiva del plan para Cuenca, muestran que, si en algún momento Gatto Sobral no simpatizaba con las posturas de Le Corbusier, al final terminó haciéndolo, entendiendo también que su proyectación tenía un mediano interés en el ámbito patrimonial (Anexo Tabla 3).

\section{Segundo momento: Estudios de casos}

Para continuar el proceso con este segundo momento, se realiza una adyacencia al plan urbano moderno, referenciando lo realizado por Muy y
Samaniego (2009), la investigación expone planes urbanos realizados en el Ecuador por motivo del terremoto de 5 de agosto de 1949; los casos analizados son:

a) Pelileo ${ }^{6}$, a decir de Muy y Samaniego (2009), en referencia al redibujo exponen que "las trazas existentes en la actualidad, se disponen de este a oeste (damero y concéntrica respectivamente), mismas que se encuentran separadas por un cruce de vías, que hace las veces de embudo y concatena a los dos Pelileo en uno" (p. 86) (Anexo Fig. 1).

Figura 1. Plano general de trazas del cantón Pelileo, Ecuador

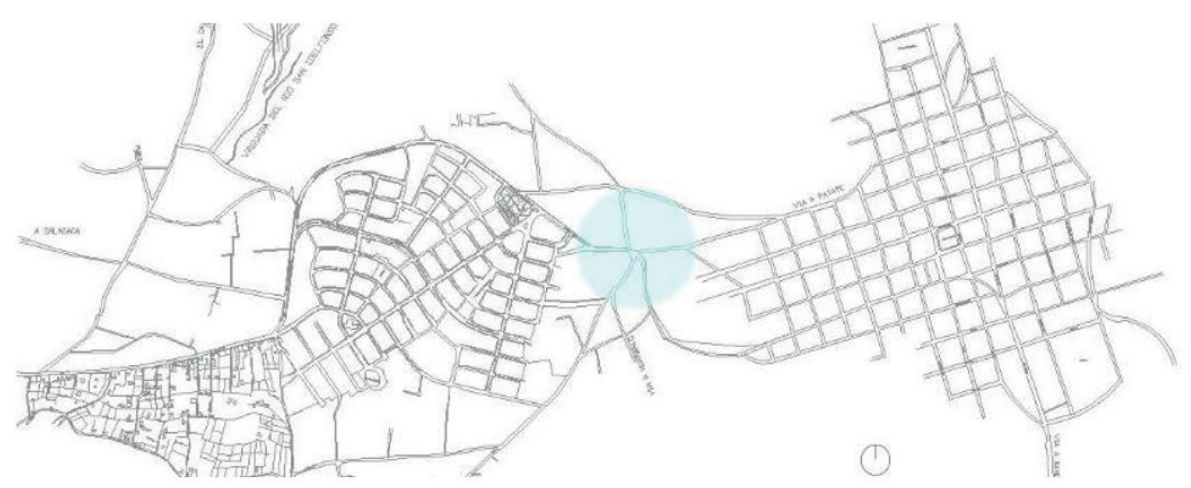

De Muy, (2009, p.86).

b) Pillaro ${ }^{6}$, a decir de Muy y Samaniego (2009), en referencia al redibujo exponen que, para identificar el origen concéntrico de la nueva traza, se parte de dos vías paralelas (sentido este - oeste) que atraviesan toda la ciudad y a la altura de la arista de un equipamiento (cyan) se propone el origen del nuevo plan. (p. 96). (Anexo Fig. 2).

\footnotetext{
${ }^{5}$ Muy, N. (2009). Influencia del arquitecto Gilberto Gatto Sobral en la concepción urbana moderna de la ciudad de Cuenca. Biblioteca de Maestría de Proyectos Arquitectónicos. Facultad de Arquitectura. Universidad de Cuenca., p. 86, p. 96, p. 91. Mapa Pelileo Anexo 4, p. 101. Mapa Pelileo Anexo 4.

${ }^{6}$ Muy, N. (2009). Influencia del arquitecto Gilberto Gatto Sobral en la concepción urbana moderna de la ciudad de Cuenca. Biblioteca de Maestría de Proyectos Arquitectónicos. Facultad de Arquitectura. Universidad de Cuenca., p. 86, p. 96, p. 91. Mapa Pelileo Anexo 4, p. 101. Mapa Píllaro Anexo 5.
} 
Figura 2. Plano general de trazas del cantón Píllaro, Ecuador

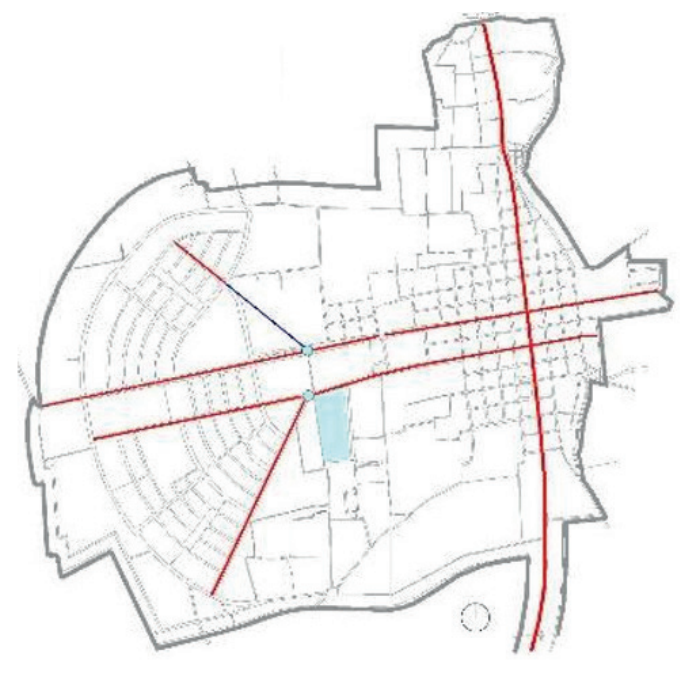

De Muy, $(2009$, p. 96).

c) Con base en todo lo anterior, se realiza un acercamiento al Plan Piloto para Bogotá desarrollado por Le Corbusier. Como particularidad se indica que los planes de Bogotá y Cuenca, se desarrollan de manera paralela y que tienen características similares como su altitud, así, la primera bordea los 2.600 msnm., mientras que la segunda esta por los 2.560 msnm., respectivamente; otra característica símil es que las dos están atravesadas por cañadas (cause con caudal temporal), quebradas y ríos, incluso por ciertas particularidades paisajísticas. Como análisis en el caso Bogotá, se toma como referencia la múlti- ple información que muestra la página de internet: Le Corbusier en Bogotá7. Aquí se explica, que él, condiciona su planificación con el reconocimiento de los valores, históricos, geográficos y topográficos; luego parte de un criterio de zonificación, para emplazar las cuatro funciones básicas: habitar, trabajar, recrear, circular; seguidamente identifica dos vías existentes, Av. Cundinamarca y calle 13, la primera divide a la ciudad en dirección Norte-Sur, la segunda es perpendicular a la primera y divide a la ciudad en sentido Este-Oeste; es a través de estas longitudes que el Plan se establece (Anexo Fig. 3). 
Figura 3. Plan piloto para Bogotá de Le Corbusier

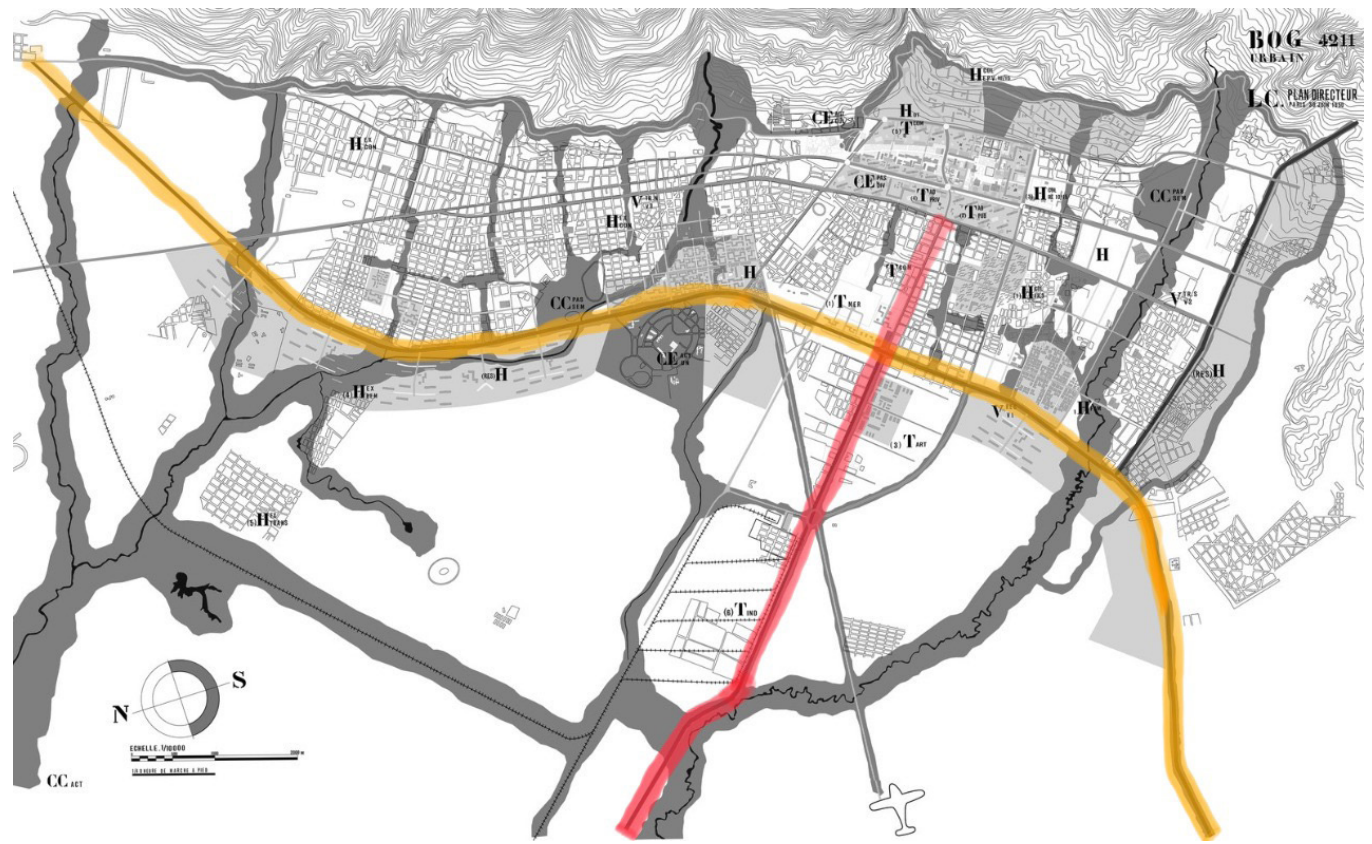

De O'byrne Orozco, (2008, http://www. lecorbusierenbogota.com, Le Corbusier en Bogotá: Los Libros, Precisiones en torno al Plan Director, Tomo 2, Tercera Parte).

Lo que nos da este segundo momento, sin lugar a dudas es el valor y respeto que muestran las intervenciones sobre la preexistencia en distintos ámbitos, es por eso que surgen dudas, en el sentido de que el plan urbano para Cuenca guarda otros criterios que no han sido revelados.

\section{Tercer momento: Investigación exploratoria}

El tercer momento surge como a manera de conclusión de los dos anteriores, lo que se describe a continuación es una exploración con base en la investigación de Muy y Samaniego (2009), con respecto al redibujo del anteproyecto del plan regulador de 1947. Para visibilizar las acciones tomadas sobre este, se numeran (en el redibujo) a los círculos entre negros, y azul del "1" al "13" y a las líneas rojas (vías) con literales de la "A" a la "G", los dos en sentido horario Este- Oeste (Anexo Fig. 4). 
Figura 4. Gráfico Explicativo de Redibujos y Proporciones Áureas en base al plan regulador para Cuenca de Gilberto Gatto Sobral



El proceso inicia tomando como punto de partida la plaza central (hoy parque Calderón), cuyas dimensiones originales (91.0795 m x $92.7665 \mathrm{~m}$ ) determinan que el equipamiento tiende a un cuadrado perfecto, es por esto que se decide actualizar su geometría a la proporción áurea; esta modificación (91.0795 m x $91.0795 \mathrm{~m}$ ) resulta en una diferencia de $1.687 \mathrm{~m}$., con respecto a la primera. Establecido el módulo de partida, se procede a incrementar hacia fuera del primer cuadrado, en distancia de $91.0795 \mathrm{~m}$., hasta 36 repeticiones; a continuación, se sobrepone el bloque de cuadrados sobre el re dibujo del plan regulador anulando los 40 de inclinación en dirección Este hasta alcanzar el norte y sumando $7^{\circ}$ de inclinación en sentido Noroeste, por supuesto considerando su origen rotatorio, al punto medio del cuadrado original. Esta acción geométrica muestra coincidencias con respecto a los puntos de intersección vial. Con el procedimiento anterior se asume parcialmente que las intersecciones dependen del módulo original de ciudad, sin embargo, con base en las líneas de tiempo matriz dos (caso Washington D.C.), ¿qué pasa si a la disposición de los módulos cuadrados, se le aplica la misma pro- 
porción áurea, pero con el uso del pentagrama y la estrella de cinco puntas?, pues bien, la respuesta es más reveladora, ya que a la cuarta repetición del pentágono y la estrella, una de las longitudes del ala Este coincide con la inclinación que demarca la línea roja (A) que corresponde al aeropuerto; luego desde el centro del cuadrado original se proyectan cinco ejes $(a, b, c, d, e)$, hacia las puntas externas de la estrella, encontrando las siguientes relaciones: Proyección (b), con línea (A), marca la ubicación de en donde debería proyectarse el área administrativa del equipamiento. Proyección (c), con cuadrado (16), encuentra el punto o intersección (2). Proyección (d), con cuadrado (14), encuentra el punto o intersección (7) y define aproximadamente la línea (E). Proyección (e), con cuadrado (26), encuentra el punto 0 intersección (11).

Estas respuestas dan la pauta para volver a proyectar cinco ejes ( $\left.a^{\prime}, b^{\prime}, c^{\prime}, d^{\prime}, e^{\prime}\right)$ desde el mismo centro hacia las puntas internas de la estrella, las relaciones que se encuentran son:

Proyección (b'), con cuadrados $(30,31)$, demarca aproximadamente la dirección y hasta donde tiene que proyectarse la línea (C). Proyección (e'), con cuadrados $(26,27)$, demarca aproximadamente la dirección y hasta donde tiene que proyectarse la línea $(G)$.

Con estas revelaciones, se interpreta, que el resto de intersecciones se encontrarían fraccionando a la mitad el último ángulo provocado por el origen hacia las puntas internas.

El ángulo encontrado es de $18^{\circ}$, y aunque su precisión en cuanto a coincidencias no es exacta se puede definir varias intersecciones relevantes como los puntos 4, 6, 8, y las líneas D (actual Av. Solano), y F (longitud hasta el punto 8); como caso especial se menciona a la línea (B), que resulta paralela a la (C) y su intersección con el cuadrado (30) limita su longitud y encuentra al punto (1). Abajo se explican las relaciones con el ángulo de 180:
Proyección (a', c), con cuadrado (21), encuentra el punto o intersección (4). Proyección (d, $\left.a^{\prime}\right)$, con cuadrado (11), encuentra el punto o intersección (6), y con cuadrados $(26,27)$ limita el crecimiento de (D). Proyección $\left(e^{\prime}, d\right)$, no es tan precisa, sin embargo, con cuadrados $(16,17)$, se aproxima a delimitar el punto 0 intersección (8), que también limita el crecimiento de (F). El procedimiento dado, deja aparentemente sueltos únicamente a los puntos 3, 5, 9, $10,12,13$, sin posibilidad de intersecar la geometría modular con uno de los ejes, sin embargo, hay que aclarar que todos estos números tienen una relación aproximada con los módulos cuadrados, la diferencia se coloca entre paréntesis:

El centro del punto 3 coincide con el cuadrado número 19 (1.87 m). El centro del punto 5 coincide con el cuadrado número $18(11.90 \mathrm{~m})$. El centro del punto 9 coincide con el eje de los cuadrados 18 y 17 (6.51 m). El contorno del punto 10 coincide con la del cuadrado 36 (9.02 m). El centro del punto 12 coincide con el contorno del cuadrado 29 (3.07 m). El centro del punto 13 coincide con el cuadrado número 16 (5.09 m). 


\section{Conclusión}

En relación al módulo como ente original de delimitación (el criterio clásico como proporción):

Que la ciudad proyectada por el Arq. Gatto Sobral, plantea el criterio de tomar a la plaza fundacional, como módulo originario de la nueva ciudad, así lo demuestra la dimensión aurea de 91.0795 metros, misma que repetida hasta 36 veces, delimita la planificación, y pone en valor a toda la ciudad histórica. Dentro del mismo criterio provoca la inclusión del pentágono, la estrella de cinco puntas y las diagonales (áureos todos), con el fin de explorar y emplazar los componentes internos de ciudad con criterios de orden.

En relación a la valoración esencial del proceso histórico (traza originaria como medio de crecimiento urbano):

Que el anteproyecto del plan regulador fusiona la ciudad primera con la de 1947, decantando criterios, como, la de provocar una traza concéntrica teniendo como base la traza fundacional, también el usar bordes como el río Tomebamba y el barranco como ligaduras físicas entre lo nuevo y lo preexistente, en relación a esto, si redujéramos la escala y actuáramos en el aspecto de arquitectura como intervención, a tenor De Gracia冬 (1997, p.187), sería una relación de exclusión, solucionada por medio de un conector específico, 0, una sutura llamada "poché". El criterio de ampliación de la traza hacia el este del Centro Histórico, presenta similitudes a lo planteado por Ildefonso Cerdá en Barcelona (1859), y también con lo realizado en Píllaro (1949), las dos ciudades marcan su intervención de ampliación y ensanche a partir de la preexistencia. En el caso de Cuenca se basa en una proyección diagonal aurea hacia el este, que parte de la calle Sur que delimita el cuadrado fundacional y luego traza una paralela a dos cuadras de distancia, a partir de esta actuación se plantea una nueva forma urbana (Anexo Fig. 5).

${ }^{8}$ De Gracia, F. (1992). Construir en lo construido. La arquitectura como modificación. Madrid: Editorial Nerea. 
Figura 5. Gráfico Explicativo de Redibujos y Proporciones Áureas sobre puesto al plan regulador para Cuenca de Gilberto Gatto Sobral

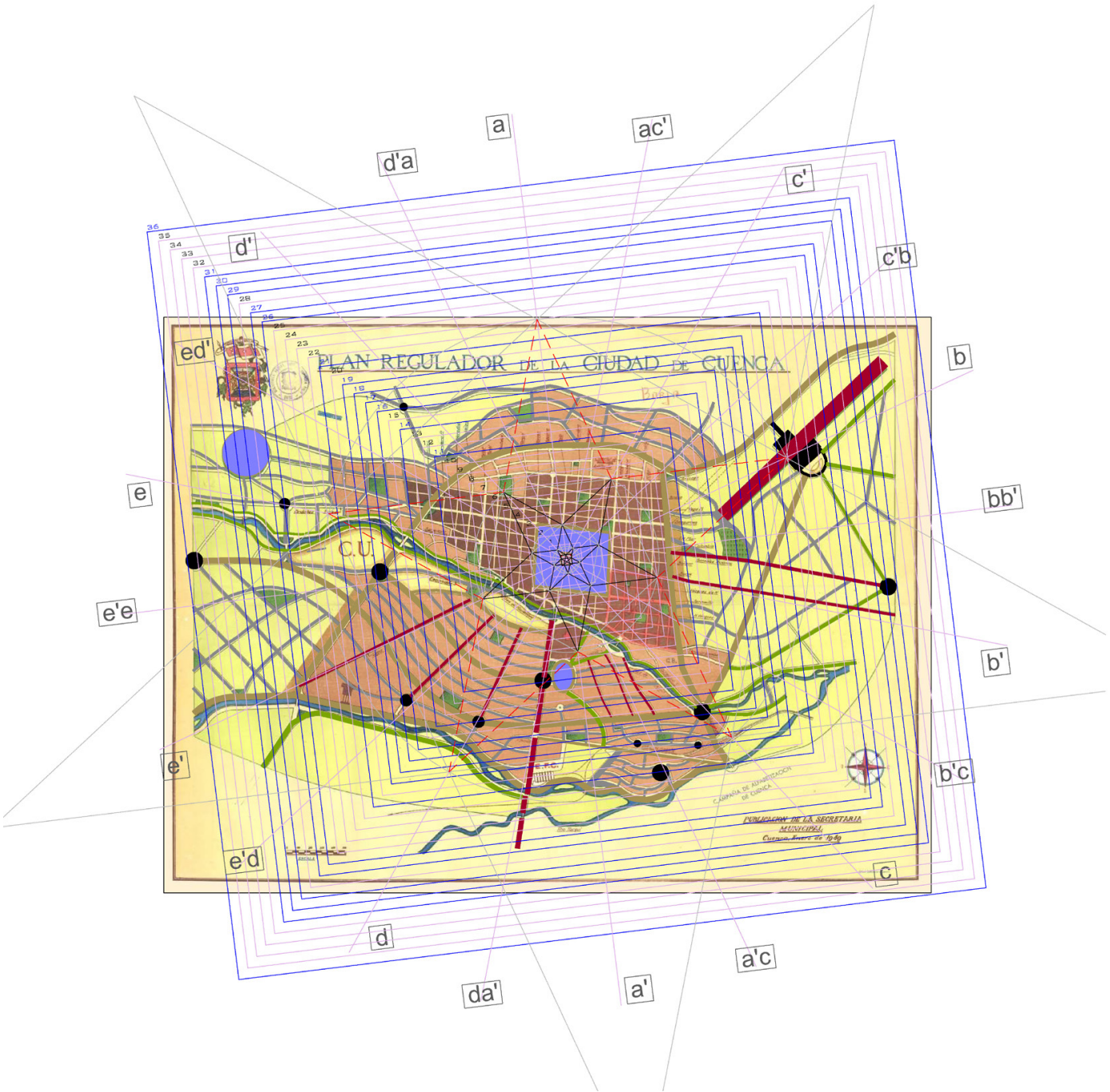

La condición de la forma urbana de la Cuenca de 1947. Que a tenor de Muy y Samaniego, (2009), Al haber verificado que la Cuenca del Plan de una u otra manera se ha servido de los criterios dados en un ritmo histórico, se considera entonces que el proceso de hacer arquitectura 0 urbanismo no se rige a la imaginación de su creador, sino al conocimiento histórico de su formación, nada es reconocido si es que no hay una aplicación coherente de los conocimientos preexistentes (p. 285). 
Al respecto Helio Piñón", en otro axioma de la forma moderna sentencia "La materia prima de la arquitectura es la propia arquitectura, no las ideas". Que a tenor de Muy y Samaniego, (2009). "La aplicación de la nueva traza establece el paso de la manzana al bloque, porque sus proyecciones de vías conforman bandejas de gran área, superando a la manzana del casco histórico en un $120 \%$ (en sus bloques mínimos)" (p. 286). Según la Unesco (2003) ${ }^{10}$, el Patrimonio Cultural inmaterial responde a "expresiones que se han transmitido de generación en generación, han evolucionado en respuesta a su entorno y contribuyen a infundirnos un sentimiento de identidad y continuidad" (p.5). Partiendo de esta consideración se logra entender, que, la ciudad de Cuenca en 1947 fue intervenida de manera contextual en la disciplina de la arquitectura. Su visión nunca irrespeta, la altura máxima que alcanza la ciudad histórica por medio de la catedral, la cual, para el momento del plan era considerada un hito arquitectónico, que permitía identificar la ubicación de la preexistencia histórica desde cualquiera de sus dos circunvalaciones (Norte, Sur), ubicadas así por el respeto a la topografía del lugar. Han sido revelados criterios de intervención, basados en condiciones intrínsecas de un trabajo técnico, intuitivo y racional, que no aplica únicamente lo que le dice la historia en función de la ciudad jardín, o la inclusión de las cuatro funciones del urbanismo moderno, sino que además la ordena bajo los principios de una antigüedad clásica - renacentista, juntando el ámbito terrenal (hombre) con el ámbito inmaterial áureo (Dios), como lo hizo Da Vinci con su hombre de Vitrubio.

\section{Referencias}

De Gracia, F. (1992). Construir en lo construido. La arquitectura como modificación. Editorial Nerea. Maldonado, C. (1991). Gilberto Gatto Sobral en la arquitectura ecuatoriana. Revista Arquitectura No. 261. Sociedad de Arquitectos de Uruguay. Editorial Escuelas Profesionales Talleres Don Bosco.

Piñón, H. (2010). Arquitectura de la ciudad moderna. Ediciones UPC, pp. 254.

Real Academia Española. (2001). Diccionario de la lengua española (23.a ed.). https://dle.rae.es/?i$\mathrm{d}=$ BK4MHWL

Real Academia Española.(2001). Diccionario de la lengua española (23.a ed.). https://dle.rae.es/?i$d=$ DsDPysR

Real Academia Española.(2001). Diccionario de la lengua española (23.a ed.). https://dle.rae.es/?i$d=$ LdvyG1W

Samper Gnecco, G. (1997). Recinto Urbano "la humanización de la ciudad". Fondo Editorial Escala. UNESCO. (2003). United Nations Educational Scientific and Cultural Organization. Intangible

Cultural Heritage. https://ich.unesco.org/doc/src/01851-ES.pdf

\footnotetext{
${ }^{9}$ Piñón, H. (2010). Arquitectura de la ciudad moderna. Barcelona, España: Ediciones UPC, pp. 254.

${ }^{10}$ UNESCO. (2003). United Nations Educational Scientific and Cultural Organization. Intangible Cultural Heritage. Recuperado de: https://ich.unesco.org/doc/src/01851-ES.pdf.
} 


\section{Figuras}

Figura 1. Muy, N. (2009). Influencia del arquitecto Gilberto Gatto Sobral en la concepción urbana moderna de la ciudad de Cuenca. Facultad de Arquitectura (Biblioteca de Maestría de Proyectos Arquitectónicos), Universidad de Cuenca p. 86, p. 96, p. 91. Mapa Pelileo anexo 4, p. 101. Mapa Pelileo anexo 4.

Figura 2. Muy, N. (2009). Influencia del arquitecto Gilberto Gatto Sobral en la concepción urbana moderna de la ciudad de Cuenca. Biblioteca de Maestría de Proyectos Arquitectónicos. Facultad de Arquitectura. Universidad de Cuenca., p. 86, p. 96, p. 91. Mapa Pelileo anexo 4, p. 101. Mapa Píllaro. anexo 5.

\section{Redibujos}

Figura 4 y 5. Muy N. y Samaniego P. (2019). "Redibujos y Proporción Aurea", anexos 7,8,9. Cuenca. Figura 5. O'byrne Orozco, M. (2008). "Le Corbusier en Bogotá. Bogotá, Colombia". http:// www.lecorbusierenbogota.com 